\title{
LA DINÁMICA DE LAS RELACIONES ENTRE INVESTIGADORES Y PROFESIONALES CON MOTIVO DE UNA INVESTIGACIÓN CONTRACTUAL
}

https://doi.org/10.17979/relaso.2011.1.1.1195

\section{Yvonne Guichard-Claudic}

Maître de conférences en sociologie

Université de Bretagne Occidentale (Brest)

Atelier de Recherche Sociologique (ARS - EA 31 49)

Yvonne.Guichard-Claudic@univ-brest.fr

\section{Resumen}

El enfoque del artículo es principalmente metodológico. Pone una mirada reflexiva sobre las relaciones del sociólogo con los socios institucionales que solicitan su peritaje con motivo de un contrato de investigación. Se funda sobre una investigación llevada en 2009-2010 para el Consejo general de Finistère sobre la importancia de los servicios públicos en los recursos movilizados por los padres en caso de dificultades educativas (386 cuestionarios a allegados, 30 entrevistas con madres y padres, 18 entrevistas con profesionales). Se trataba de recoger su experiencia, su punto de vista con el fin de mejorar su acompañamiento en caso de dificultades.

Palabras clave: combinación de metodologias, relaciones sociólogo-comanditarios, apoyo parental

\section{Résumé}

Le propos de cet article est d'abord méthodologique. Il pose un regard réflexif sur les rapports qu'entretient le sociologue avec les partenaires institutionnels qui sollicitent son expertise à l'occasion d'un contrat de recherche. Il se fonde sur une enquête menée en 2009-2010 pour le Conseil Général du Finistère sur la place des services publics dans les ressources mobilisées par les parents en cas de difficultés éducatives (386 questionnaires parents, 30 entretiens avec des parents, 18 entretiens avec des professionnels). Il s'agissait d'appréhender l'expérience de parents afin d'améliorer leur accompagnement en cas de difficultés. Mots-clés : combinaison de méthodologies, relations sociologuecommanditaires, soutien parental 


\section{Summary}

The purpose of this paper is primarily methodological. It is a reflective look on the sociologist's relations with institutional partners who seek his expertise on the occasion of a research contract. It is based on a survey conducted in 2009-2010 to the General Council of Finistère on the role of public services in the resources mobilized by the parents in case of educational difficulties (386 questionnaires parents, 86 and 30 interviews with parents, 18 interviews with professionals). This was to understand the experience of parents to improve their support in case of difficulties.

Keywords: combination of methodologies, relations sociologistsponsors, parental support

\section{Marco teórico}

Basarse en la experiencia de una investigación contractual llevada para el Consejo general del Finistère ${ }^{1}$ para poner una mirada reflexiva sobre las relaciones del sociólogo con los socios institucionales que solicitan su peritaje con motivo de un contrato de investigación, tal es el enfoque expuesto aquí.

En efecto, practicar la investigación contractual, responder a licitaciones que predefinen de un cierto modo nuestras preguntas, lo que puede aparecer contrario a una postura científica que busca el conocimiento por sí mismo, de una manera desinteresadamente para aumentar el conocimiento y no para un propósito práctico, es una de las características de nuestra disciplina que no se encuentra por ejemplo en historia o en literatura. Sin embargo, la problemática de la respuesta de la sociología a la demanda social no es nueva; es constitutiva de la

1 El Consejo general es una asamblea elegida por los departamentos franceses. Tiene varias misiones: acciones sociales, prevención y protección de los niños y jóvenes, la ayuda a las personas edades, la organización del espacio y del equipo al nivel departamental, el mantenimiento de los colegios, los museos etc. etc... 
emergencia de la disciplina. "Consideramos que nuestras investigaciones no valen una hora de pena si debian tener sólo un interés especulativo ", declaraba Emile Durkheim en una obra fundadora ${ }^{2}$. Ayudar a comprender los problemas que constituyen la trama de la vida cotidiana de numerosos individuos y alumbrar a los " responsables " son objetivos clásicos del trabajo sociológico. Sin embargo, este enfoque no va de sí. Haremos aquí la apuesta que si el investigador respeta los criterios de rigor específicos de la epistemología de nuestra disciplina y si sus socios le concedan la confianza, su encuentro puede ser portador de conocimiento para analizar los temas de actualidad y fundar, al menos en parte, elementos de política o más modestamente, mejorar ciertas prácticas en el futuro.

El artículo se funda sobre una investigación llevada en 20092010 para el Consejo general del Finistère ${ }^{3}$ sobre la importancia de los servicios públicos en los recursos movilizados por las madres y padres en caso de dificultades educativas ${ }^{4}$. Se trataba de aprehender su experiencia, de recoger su punto de vista con el fin de mejorar su acompañamiento en caso de dificultades.

Hay que referir la investigación al contexto social y político: las dificultades que pueden encontrar algunos padres en su papel educativo, pero también la inquietud en relación con las transformaciones de la familia $y$ sus consecuencias en el comportamiento social de los jóvenes, condujeron los poderes públicos a poner en ejecución acciones de apoyo paterno. Esta cuestión del apoyo a las madres y padres constituya hoy un elemento fuerte de la reflexión y de las intervenciones de las administraciones en materia de salud, de justicia, de trabajo social y de familia. Convendría trabajar con las familias, asociarlas con la acción de los profesionales, considerarlas como socios.

2 DURKHEIM Emile, De la division du travail social, Paris, PUF, 1986 para una edición recente.

${ }^{3}$ El Finistère es un departamento de una región francesa, la Bretagne.

${ }^{4}$ GUICHARD-CLAUDIC Yvonne (dir ${ }^{\circ}$ ), BIHANNIC Lise, avec la collaboration de CHARRIER Gilda, DEROFF Marie-Laure et POTIN Emilie, La place des services publics dans les ressources mobilisées par les parents en cas de difficultés éducatives. Le point de vue des parents finistériens, Rapport pour le Conseil Général du Finistère, Brest, noviembre 2010. 
Sin embargo, esta noción de apoyo a la parentalitad no constituye una base de acción claramente delimitada. Es subtendida por lógicas complejas (Avenel, 20035) que van de la preocupación de los poderes públicos de proteger al niño, particularmente de padres desfallecientes, para mantener el orden público hasta la valorización de las tareas educativas y de la responsabilidad de los padres que intentan, a través de paradas exteriores, de llevar a cabo las tareas paternas que les incumben. Es este movimiento doble de incriminación-valorización (Boisson, Verjus, 20046) de la función de los padres que explica que el acompañamiento de los madres y padres se expresa, según los casos, más sobre el lado de la limitación o sobre el del apoyo.

Además, medidas de apoyo parental son muy diversas, dispersas geográficamente y no necesariamente articuladas. Sabemos muy poco sobre el conocimiento que tienen los padres y madres de las distintas acciones pero la información a las familias, especialmente los más desfavorecidas, debe ser un componente esencial de cualquier política de prevención. También se puede legitimamente preguntarse qué promueve o frena el uso de estos servicios, ya que los servicios son identificados por los padres y madres como capaces de ayudarles en caso de dificultades educativas. Finalmente las opiniones de los padres y madres directamente afectados por una medida de apoyo a la crianza de los hijos, ya sea primaria, secundaria o terciaria ${ }^{7}$, siguen siendo relativamente desconocidas.

5 AVENEL Cyprien, "La relation aux aides sociales " du point de vue " des bénéficiaires ", Recherches et prévisions, $\mathrm{N}^{\circ}$ 72, junio 2003, p. 37-52.

6 BOISSON Marine, VERJUS Anne, La parentalité, une action de citoyenneté. Une synthèse des travaux récents sur le lien familial et la fonction parentale (1993-2004), CNAF, Dossier d'étude n 62, noviembre 2004.

$7 \mathrm{Si}$ se ajusta a la definición de la prevención primaria utilizada por la OMS (Organización Mundial de la Salud) para el campo de la protección de los niños, todos los" actos destinados a reducir la incidencia de las dificultades de una población antes que parecen " contribuyen a este objetivo. (...) La prevención secundaria incluye "las acciones destinadas a reducir la prevalencia de las dificultades de una población. "(...) La prevención terciaria pretende reconstituir los recursos y / o limitar los efectos de desestructuración en relación con una crisis o dificultad. "" Los actores de la 
El deseo de entender con mayor precisión la experiencia de los padres y madres sobre estos temas condujo el Consejo General del Finistère a presentar una licitación para un estudio dedicado al acceso de la población a los servicios de protección infantil. Es a la licitación que respondimos y para la que hemos sido retenidos.

Las colaboraciones forjadas a esta ocasión fueron más numerosas, más cerca en el tiempo y más ancladas en la duración que lo que habíamos conocido en otras investigaciones. Es por eso que nos condujeron a cuestionar las interacciones entre el sociólogo y su socio comanditario. Pues la intención de este artículo es más reflejar sobre un punto de metodologia que presentar los resultados de este estudio.

Tres tiempos, que respetan más o menos la cronología de la investigación organizarán el propósito: el primer tiempo se ocupará del desarrollo de los interrogatorios y la metodología utilizada en las diferentes etapas de la investigación; el segundo concernirá a la lectura hecha por los profesionales de nuestros trabajos. Aquí, es el encuentro del conocimiento que viene de las prácticas profesionales de unos y otros que está en juego, con los azares eventuales y las incomprensiones que pueden sobrevenir, pero también a veces nuevas ideas sobre nuestros resultados. En un tercer tiempo por fin, es el anclaje de estos trabajos en un territorio y en una temporalidad de duración más larga que será contemplada.

\section{Una metodologia cualitativa con el apoyo de una amplia colaboración}

Descomponer y reconstruir la demanda en las categorias y con los métodos que son las de nuestra disciplina, tratando de responder lo mejor posible a la demanda que nos fui exprimida: así es como podemos resumir nuestro enfoque.

Empecemos, en pocas palabras, con la pregunta de este estudio. Los objetivos eran los siguientes:

protección del niño en Finistère ", CG29, Dirección Infancia-familia, $09 / 10 / 2008$. 
- Identificar las situaciones y vivencias de los padres y madres que consideran como dificiles en la educación de sus hijos

- Evaluar su conocimiento en las distintas formas de apoyo propuestas en caso de dificultades educativas: ¿es este conocimiento muy fragmentario o concierna un gran parte de la gama de los servicios propuestos y de los actores involucrados? ¿Cómo obtuvieron la información que detienen? ¿Qué nos dice esto sobre la accesibilidad de los servicios?

- Identificar las representaciones de estos servicios, desde que tienen un conocimiento, aunque se aproximado: ¿tienen una imagen estereotipada y negativa o los consideran como una solución posible para sus dificultades? ¿Cuál representación tienen de las familias, de los medios, que, según ellos, recurren a estos servicios? ¿Cuál es el origen de estas representaciones?

- Conocer mejor a las familias que reciben ayuda en la protección del niño o el apoyo a la maternidad: ¿cuáles son sus características sociales? ¿Cuál es el camino que les ha conducido a beneficiarse de ellos? ¿Cómo evalúan la experiencia que tienen de la medida?

- Identificar los factores que impiden o facilitan el recurso a los servicios, para ayudar la definición de acciones que mejoren la accesibilidad a los servicios

- Al comparar estas representaciones de la población global de los padres y madres del departamento del Finistère, la experiencia del grupo más reducido de padres "ayudados" y la de los profesionales y otros actores de la protección infantil, identificar los factores que frenan o al contrario favorecen la pregunta de ayuda en caso de dificultades, con el fin de identificar las acciones para mejorar el acceso a los servicios.

La licitación llevaba una atención particular en la metodología para realizar este estudio. Proponía un enfoque relativamente preciso, que hemos respetamos en parte, pero que hemos reformulado en una dirección que nos parecía más conforme con los objetivos perseguidos. Tres fases estuvieron previstas: 
Fase 1 (la licitación): una parte del estudio deberá referirse en una muestra representativa de padres y madres que encontrarán o habrán encontrado dificultades educativas, una necesidad de apoyo de la parentalitad. La muestra, la investigación y los resultados serán realizados y explotados por el prestatario, después de validación por el Consejo general de Finistère.

Por la primera fase, la demanda llevaba sobre una muestra representativa de madres y padres que encontraban o habian encontrado dificultades educativas, una necesidad de apoyo de la parentalitad. Esta formulación sugería la realización de un cuestionario cerca de una larga población de encuesta, que sea representativa de la población de todos los padres y madres del departamento. Este deseo nos parecía contradictorio con la definición que la licitación daba de las dificultades educativas. "Las " dificultades educativas " de las cuales tratamos aquí cobren una realidad dificil a caracterizar precisamente porque fluctúa según las situaciones, según los recursos movilizables por los padres y madres. Este estudio se interesa a las problemáticas encontradas por los padres y madres en la educación de su hijo, que se sienten "abrumados" por la situación. Aquí es el sentimiento de los padres y madres que importa, más que la identificación objetiva de la situación. "

Estamos tratando aquí con una definición cualitativa del concepto de "dificultades educativas", basada en el sentimiento de las madres y padres que se sienten "abrumados" por la situación más que en la identificación objetiva de las dificultades. Para entender las percepciones y experiencias subjetivas, un enfoque comprensivo y exploratorio parecía más apropiado que un cuestionario estandarizado. Pues es lo que propusimos.

La primera etapa consistió en un estudio exploratorio que pretendia definir la gama de las situaciones vividas como problemáticas por los padres y madres, fundándose sobre la hipótesis que la expresión " dificultades educativas " recubre una gran variedad de situaciones objetivas y subjetivas. Se trató, a este estadio del estudio, de dirigirse hacia toda la población de 
padres y madres con el fin de cercar las dificultades, tan bien "ordinarias" como más específicas, que pueden encontrar.

No es fácil encontrar a los padres desde la entrada "dificultades" porque no es una entrada gratificante para el investigado. Pues imaginemos un protocolo que nos permitiria encontrar cara a cara una centena de padres y madres. Para hacerlo, y con el fin de realizar entrevistas sobre el tema, nos pusimos en contacto con aproximadamente 2600 padres y madres desde los establecimientos escolares donde son escolarizados sus niños (escuela maternal y primaria, colegio, liceo, general y profesional). Los establecimientos escolares han sido escogidos en municipios de todo el departamento.

La implantación de la escuela constituyó otro criterio de diversificación: tamaño de la ciudad y del establecimiento, medio rural o urbano, escuela de centro ciudad o de ZEP8 ... Esta entrada nos pareció ser la que permitía tocar a todas las familias y cubrir todas las edades de los niños o de los jóvenes. A través de los jefes de establecimientos que aceptaron colaborar en la investigación, les enviamos un pequeño cuestionario que pretendia recoger un cierto número de respuestas en nuestro tema. El cuestionario contenía un talón para regresar si la persona aceptaba de concedernos una entrevista. E1 procedimiento se reveló bastante productivo ya que recibimos 368 respuestas, es decir una tasa de respuesta de $15 \%$; sobre estos 368 que han respondido, una centena de personas aceptaba encontrarnos; en resumen, para quedarse en los limites temporales de la investigación, son 86 entrevistas que han sido realizadas en esta primera fase.

Fase 2 (la licitación): Una parte del estudio tendrá que centrarse más específicamente en una muestra de madres y padres concernidos por una medida de protección del niño o que reciben apoyo parental.

En una secunda etape (fase 2), la investigación se centró en la situación de las madres y padres gozando de un acompañamiento en protección de la infancia o de apoyo parental.

8 Zona de Educación Prioritaria. 
Inicialmente habiamos planeado ponernos en contacto con 200 madres y padres para que respondan a un cuestionario, es decir 100 concernidos por una medida de protección del niño y 100 beneficiarios de una forma de apoyo parental. Lo previsto era que el Observatorio de la protección del niño, nuestro comanditario del Consejo general del Finistère, nos proporcione la mitad de estos contactos. Incumbía al equipo de investigación de ponerse en contacto con un conjunto de estructuras para encontrar a los 100 otros padres.

El proceso iniciado por el Observatorio cerca de los territorios de acción social no tuvo éxito y sólo 8 padres y madres fueron reclutados de esta forma y su perfil no correspondia realmente a las situaciones buscadas; las mamás que frecuentaban el centro de protección materno-infantil para seguimientos de lactancia o de curva de peso constituían la mitad de este grupo.

De acuerdo con el Observatorio, hemos revisado la metodología inicialmente prevista: el equipo de investigación propuso encontrar a profesionales de estructuras que intervenian en protección infantil o en apoyo parental para iniciar un enfoque monográfico: una entrevista con uno o varios profesionales de la estructura seguida de 4 o 5 entrevistas con padres y madres usuarios de los servicios de esta estructura. La idea era repartir las entrevistas entre los tres niveles de prevención: primaria, secundaria y terciaria. Recordemos que la prevención primaria tiene como objetivo disminuir la incidencia de las dificultades de una población antes de que éstas aparezcan, la prevención secundaria pretende reducir la prevalencia de las dificultades y la prevención terciaria reconstituir recursos $\mathrm{y} \backslash \mathrm{o}$ limitar los efectos de desestructuración en relación con una crisis o una dificultad ${ }^{9}$. En esta óptica, el equipo solicitó 24 estructuras que se encargan de uno o varios de estas misiones con el fin, en primer lugar de encontrar a uno o varios profesionales de terreno, en contacto con los padres y madres en la estructura, y de hablar de su práctica, por otra parte que este profesional oriente a los investigadores hacia algunas situaciones de madres o padres y les ayude a obtener su aprobación para la realización de una entrevista sobre su experiencia parental. Las estructuras se mostraron

${ }_{9}^{9}$ Definición de la OMS (Organización Mundial de la Salud). 
generalmente abiertas a nuestro enfoque, pero al final son 18 estructuras que nos concedieron una entrevista, con la persona responsable, a menudo con su equipo. Las estructuras que nos pusieron en contacto con padres y madres nos permitieron cada vez realizar 1 a 2 conversaciones, excepcionalmente 3. En resumen, sólo 37 padres y madres fueron contactados de ese modo; ciertas estructuras llegaron a ponernos en contacto con padres y unas no eran en situación de hacerlo (por ejemplo estructuras donde la identidad de los padres no es surtida). Sobre estos 37 contactos, 30 acabaron en una entrevista, ciertas personas por haber librado del último minuto, rechazando finalmente la entrevista o anulándola para reportarla a una fecha incompatible con los imperativos de la investigación.

Se puede observar, los imprevistos de la investigación nos condujeron a adaptar nuestra metodología. Cada vez, las opciones se realizaron de acuerdo con nuestro comanditario, lo que no era evidente porque todavía no estábamos seguros de poder llevar a cabo nuestros objetivos. Una gran sinceridad era necesaria, y sin embargo había que permanecer creíble. Es verdad que el fracaso del Consejo general en lo que concernía a la implicación de sus profesionales de los territorios de acción social permitía relativizar nuestras propias dificultades. Frente a los obstáculos encontrados para acceder a un número suficiente de padres, la elección entrevistas en profundidad, a carácter biográfico, preferentemente a un cuestionario estandarizado, se imponía. Estas entrevistas, con una duración de 30 minutos a 2 horas según los casos, fueron a menudo muy ricos y nos permitieron acceder a situaciones diversas, a veces muy dificiles cuando se trataba de prevención secundaria y sobre todo terciaria. Se celebraron en el domicilio de la persona o en otro lugar de su elección.

La atención llevada por los miembros del servicio infancia del Consejo General a las cuestiones metodológicas no desdijo y encontró un prolongamiento directamente operativa después. En efecto, la necesidad de escuchar la palabra de los padres y madres fue reafirmada por el ministerio, que pedía a los servicios reunir a padres y hacerlos dialogar; nuestras interlocutoras 
fueron alertadas por las dificultades que podría ser la organización, en un corto periodo de tiempo, de tales dispositivos. Pues nos pidieron redactar una nota metodológica para explicar la complejidad de nuestro enfoque.

Fase 3 (licitación): una parte del estudio permitirá destacar la opinión de los profesionales, los elegidos y los representantes de usuarios interesados en el tema para poner en perspectiva lo que identifican como frenos o facilitadores en el acceso a los servicios. El Observatorio departamental de la protección del niño podrá poner en disposición del prestatario la red de los socios así como todos espacios colectivos necesarios de trabajo, particularmente en el contexto de los trabajos preparatorios al esquema de la infancia y de la juventud.

En la primavera de 2010, tres mediodías han sido dedicados a reuniones con profesionales de los servicios del apoyo a la crianza de los hijos o la protección de la infancia: representantes del proyecto educativo local de la ciudad de Brest y otros miembros de la Educación nacional, de la UDAF (Unión Departamental de las Familias), profesionales de los territorios de acción social y otros miembros de los servicios sociales del Consejo general, de la policia, una asociación de apoyo parental, educadores y psicólogos que trabajaban en hogares y centros para niños o en estructuras de seguimiento en medio abierto etc.; la paleta de las estructuras interesadas era amplia.

En estas reuniones, hemos presentado las grandes líneas del informe intermediario, que resumia los primeros resultados de la etapa 1. Esta presentación dio lugar a intercambios numerosos y a veces a reacciones vivas a los comentarios de algunos padres. Podiamos lamentar que el análisis no era más adelantado cuando estas reuniones se llevaron a cabo. Esta falta de control de los tiempos era un límite real para nosotros, aunque al mismo tiempo, nos obligó a seguir adelante. Habíamos terminado de recoger el material de la primera etapa desde dos meses y habíamos efectuado una primera exploración, estábamos colocando las condiciones de realización de la etapa siguiente, consultando las estructuras citadas en el punto precedente. En este sentido, las reuniones permitieron confrontar estos primeros 
resultados con la experiencia de los profesionales. Era interesante recoger su punto de vista a este estadio de la investigación porque nos permitió resituar ciertas declaraciones de los padres y madres en un contexto institucional que no controlábamos siempre perfectamente. Por ejemplo, la chica discapacitada de uno de los padres encontrados fue escolarizada en SEGPA (Sección de enseñanza general y profesional adaptada). Primero una asistenta social escolar y luego el jefe de los servicios médicos académicos nos señalaron que no habia que confundir enseñanza especializada, en el campo del hándicap y enseñanza adaptada; el fin del SEGPA es procurar que el alumno salga del sistema escolar con un diploma profesional. Es posible que de vez en cuando se pueda encontrar en SEGPA un niño discapacitado, con un proyecto específico como se puede encontrar este tipo de niño en la enseñanza "ordinaria" pero no es su objetivo inicial.

Las mismas interlocutoras nos informaron de los nuevos dispositivos colocados para detectar mejor la dislexia, un problema del cual numerosos padres nos habian entretenido. También fueron capaces de reflexionar y dar su opinión sobre las sugerencias y propuestas formuladas por los padres.

Por último, las reuniones nos permitieron aclarar el significado de nuestro enfoque, y los objetivos perseguidos por el Consejo general. No fue inútil precisar que en ningún caso estábamos allí para emitir un juicio sobre los servicios de los que nos hablaban los padres ni para efectuar una evaluación de su funcionamiento. En este punto, el debate se calienta a veces, como energizado por malentendidos. Producíamos la palabra de los padres en ausencia de todo juicio normativo sobre el funcionamiento de los servicios pero este enfoque puede ser dificil de entender por profesionales que consideran, a menudo con razón, hacer todo para cumplir bien su misión. Entonces aquí, interrogar a los padres y madres, es aceptar escuchar lo que tienen que decir, el modo en el que sienten subjetivamente las cosas, aunque esto no corresponde necesariamente con la experiencia de los profesionales.

Puede ser también, para lo que nos concierne, proponer una interpretación alternativa de las declaraciones, en particular las de los padres y madres de los niños internados, recogidas en la segunda etapa, en relación con el estigma muy fuerte de la que 
piensan ser objeto y el deseo de defender su identidad como padres. Puede ser insistir por fin en la necesidad de limitar las interpretaciones psicologistas y de situar las situaciones individuales en un contexto social o societal más amplio.

\section{La lectura de nuestros trabajos por los profesionales}

Ya hemos evocado la lectura de nuestros trabajos por los profesionales concerniendo a una etapa intermediaria en la restitución de los resultados. Fijémonos ahora sobre la lectura a la cual dio lugar la primera versión del informe final, y luego su versión finalizada. Teníamos un montón de datos; la restitución de nuestros resultados era muy esperada para la preparación de una reunión importante en el apoyo a los padres. Poco después de la restitución de la primera versión, dotada de una conclusión no completamente acabada, una reunión con las jefes del servicio Infancia fue organizada y estas profesionales me dieron parte de su interés en diversos aspectos del informe. Estábamos particularmente interesadas en esta reunión, porque la lectura que profesionales hacen de nuestro trabajo es considerada constituir una iluminación para decisiones de agarrar o la puesta en ejecución de orientaciones políticas, por ejemplo como parte de un nuevo depósito legislativo. Pero al mismo tiempo conocemos sólo de modo imperfecto las tareas concretas, los conocimientos y las expectativas de nuestras interlocutoras. Por otro lado, la restitución de trabajos cualitativos pone el énfasis en lógicas de acciones o de trayecto de casos particulares y no es siempre evidente de subir en generalidad desde esta base. Nos preguntábamos pues lo que nuestras interlocutoras retirarian del informe, lo que iban a criticar, lo que eventualmente habrian apreciado.

Su lectura fue muy esclarecedora; poco después, la colocación de un taller de lectura de nuestro comité científico vino para completar los aportes de esta entrevista. Este conjunto de miradas cruzadas permitió reanudar y profundizar ciertos puntos y construir una conclusión verdaderamente sintética, valorizando los puntos fuertes del estudio y sus puntos ciegos, susceptibles de realimentar nuestro interrogatorio. 


\section{¿Y después? E1 anclaje de estos trabajos $y$ de esta colaboración en la duración}

¿Hasta qué punto este tipo de trabajo es susceptible de llevar beneficios tangibles? Sin duda hay que quedar modesto acerca de su impacto. Sin embargo, una atención verdadera nos es concedida por nuestros socios. Se trata para ellos de una inversión y es deseable que tuviera beneficios. Después de este estudio, la jefe del Observatorio departamental de la protección de los niños, al principio de la licitación, organizó tres talleres de lectura con profesionales voluntarios, a quienes somos convidadas. La trama de las sesiones es la siguiente: una serie de áreas es identificada en el estudio; los socios están invitados a comentar los elementos de conocimiento que retiran del estudio en esta área y por fin se les pide identificar las implicaciones de eso para el Consejo general. Hace poco tiempo, hemos también encontrado los elegidos del Consejo general del Finistère para presentarlos los resultados de nuestro estudio y tres reuniones son previstas en el departamento para hacer lo mismo con los profesionales de los territorios de acción social.

Este método no es realmente nuevo. Emilie Potin, una estudiante de nuestro laboratorio, realizó su tesis ${ }^{10}$ sobre los trayectos de niños internados desde un gran estudio que efectuó con los mismos socios y en el sitio internet del Observatorio de la protección de los niños del departamento del Finistère (ODPE 29), se puede ver no sólo el texto completo del estudio y su síntesis pero también el resultado de los talleres de lectura que siguieron el estudio y un archivo con respecto al seguimiento que le ha dado el Consejo General.

El impulso generado alrededor de las cuestiones de crianza de los hijos va a tomar sin duda en el próximo año una vuelta nueva ya que somos solicitadas como expertas para participar en dispositivos que incluyen a los padres y madres.

Para concluir, no se trata de idealizar este tipo de colaboración, que presenta necesariamente límites de nuestro punto de vista

10 POTIN E., Enfants en danger. Enfants protégés. Enfants sécurisés? Parcours de (dé)placement (s) des enfants confiés à l'Aide sociale à l'enfance, thèse de sociologie, Brest, 2009. 
(las temporalidades de la investigación no son las de la acción política o administrativa, por ejemplo) como para nuestros interlocutores (la distancia, que es esperada de nuestro enfoque, por otro lado nos hace a veces muy prudentes y repropios a las generalizaciones tempranas). Sin embargo, puede constituir un entorno de trabajo fructuoso para los investigadores como para su socio comanditario.

Por otra parte, el interés de colaboraciones como la que acabamos de describir es tanto anclar una investigación sostenible en un área como construir relaciones con los socios institucionales que encontramos en otras investigaciones.

Repetiremos aquí las palabras de Robert Castel (200011), que subraya que para él, uno de los objetivos de la sociología debe ser comprender y encargarse de lo que le plantea problema a la gente: "esto significa que existe en toda sociedad sin duda, y en la nuestra hoy ciertamente, lo que se podría llamar unas configuraciones problemáticas, cuestiones que necesitan atención, y no sólo la atención de los científicos, porque perturban la vida social, dislocan el funcionamiento de las instituciones, amenazan de invalidación categorias enteras de sujetos sociales. Me atreveré a adelantar que la paleta de estas "configuraciones problemáticas", problemáticas porque se apena comprenderlos, y más todavía dominarlos - representa un programa (¿el programa?) privilegiado por la investigación teórica tanto como práctica en sociologia."

11 CASTEL Robert, "La sociologie et la réponse à la " demande sociale " ", Sociologie du travail, 2000, p. 281-287. 


\section{Bibliografia}

AVENEL Cyprien, "La relation aux aides sociales "du point de vue " des bénéficiaires ", Recherches et prévisions, $\mathrm{N}^{\circ} 72$, juin 2003, p. 37-52.

CASTEL Robert, "La sociologie et la réponse à la "demande sociale " ", Sociologie du travail, 2000, p. 281-287.

DURKHEIM E, préface à De la division du travail social, Paris, PUF, 1986 pour une édition récente.

GUICHARD-CLAUDIC Y. $\left(\operatorname{dir}^{\circ}\right)$, BIHANNIC L., avec la collaboration de CHARRIER G., DEROFF M-L. et POTIN E., La place des services publics dans les ressources mobilisées par les parents en cas de difficultés éducatives. Le point de vue des parents finistériens, Rapport pour le Conseil Général du Finistère, Brest, novembre 2010.

POTIN E., Enfants en danger. Enfants protégés. Enfants sécurisés? Parcours de (dé)placement (s) des enfants confiés à l'Aide sociale à l'enfance, thèse de sociologie, Brest, 2009.

\section{FORMA DE CITAR ESTE TRABAJO EN BIBLIOGRAFIAS HOW TO CITE THIS ARTICLE IN BIBLIOGRAPHIES}

Guichard-Claudic, Y (2011) : "La dinámica de las relaciones entre investigadores y profesionales con motivo de una investigación contractual", Revista Latina de Sociología, $\mathrm{n}^{\circ}$ 1: 45-60, http://revistalatinadesociologia.com, ISSN 2253-6469 